\title{
What variables including spirituality determine early sexual initiation among Thai adolescents?
}

\author{
Sasitorn Roojanavech RN ${ }^{1}$, Lina Kurdahi Badr RN²*, and John Doyle ${ }^{3}$ \\ ${ }^{1}$ Lecturer of College of Nursing, Christian University of Thailand, Thailand \\ ${ }^{2}$ Professor of School of Nursing, Azusa Pacific University and Professor Emerita, UCLA, USA \\ ${ }^{3}$ Professor of School of Nursing, Azusa Pacific University, USA
}

\begin{abstract}
Aim: To investigate the predictors of early sexual initiation (SI) among Thai adolescents.

Methods: A cross sectional design was used to assess parental and child factors that may affect early sexual initiation in 430 Thai adolescents.

Results: The prevalence of early sexual initiation (SI) was higher in Thai boys and was started at an earlier age. Early SI was associated with adolescents' self-esteem, academic performance and spirituality, family structure, parents' education, and parental monitoring. However, logistic regression noted that parental monitoring was the best predictor of early sexual initiation, followed by family structure, academic performance, and spirituality.
\end{abstract}

\section{Introduction}

Based on previous research, rates of various high risk behaviors are increasing among adolescents. Statistics from several countries have indicated that early sexual initiation (SI) has increased over the past decade. This poses a serious health concern as early SI (before the end of puberty for girls about the age of 16 and for boys about the age of 16 or 17 ) is associated with several perils, including unwanted pregnancies, cervical cancer, infertility, human immunodeficiency, virus infections, and sexually transmitted diseases [1]. Early SI has also been associated with other high risk behaviors such as drug and alcohol use, smoking, and aggression $[2,3]$. Some of these behaviors can have negative health and developmental consequences well into adulthood [4-6].

In the United States, 3 million American teenagers acquire STDs every year with more prevalence in the African American and Hispanic populations $[7,8]$. Data from Europe and the Far East have likewise noted that the age for SI has declined $[9,10]$. A study conducted across 44 countries between 2005 and 2009 found that the average age for SI is 17.8 years in the United States, Australia, Turkey and Slovakia, 16.5 years in Finland, Norway, Sweden and Denmark - and 22 years or more in Malaysia, India, Singapore and China [11]. Similarly in Thailand, norms governing sexual practices have changed substantially over the last two decades with increased acceptability of premarital sex among young women, and a trend for earlier SI in both genders [12]. Most young Thai men now have their early sexual experience with their girlfriends rather than with commercial sex workers as was the case a decade ago [13-15]. A recent survey in Thailand found a decrease in the age of SI for both sexes with no significant gender difference in sex before age 15 [15]. In addition, Thailand has the highest teenage pregnancy rates in the world [16].

Most studies to date have been conducted among adolescents in Western culture, with limited research focusing on factors that may affect early sexual initiation differently in other cultures. A few studies conducted in Thailand have found that growing up with both parents and parental monitoring were protective factors, while living apart from parents, poor family relationships, and high levels of alcohol consumption were associated with a greater likelihood of having early SI [17]. In addition, to the social and behavioral factors that have been assessed in previous studies, this study investigated the role of Buddhist spirituality as a protective factor in young Thai children which has not been reported before. Buddhism is an old religion with more than 2,500 years of history with deep roots in Thai culture shared most of the population. Buddhist children are raised to avoid harming all living beings, to be compassionate and to wish all beings well and happiness [18]. However, with the migration of rural Thai youth to the cities and increased globalization traditional and spiritual values being are being lost or not valued [19].

Understanding the factors associated with initiation of SI is vital for designing and delivering interventions for Thai youth [17]. Previous research has emphasized the complex nature of adolescent sexual behavior and the importance of examining multiple risk factors across different contexts [20]. It was assumed in this study that the initiation of early sexual behavior is a decision made by the adolescent that is affected by both protective factors and non-protective factors. The theoretical framework used for this study, is a holistic view of youth development [21] which theorizes a complex interaction between the child and contextual factors such as the interaction between the family, the environment and the child. In light of growing evidence that several

Correspondence to: Lina Kurdahi Badr RN, Professor of School of Nursing, Azusa Pacific University and Professor Emerita, UCLA, 701 E foothill Ave, Azusa Ca 91702, USA, Tel: +310 341-3143; Fax: 626-815-6000; E-mail: 1badr@apu.edu

Key words: early sexual initiation, spirituality, Thai adolescents

Received: January 07, 2016; Accepted: February 08, 2016; Published: February 11, 2016 
factors can play important roles in adolescents' sexual decision making, the factors assessed in this study were family variables (structure, monitoring and education, and adolescent variables (grades, selfesteem, and spirituality).

Family variables: In most studies, the family system has been shown to be among the most influential in ameliorating risk behaviors, including substance use and sexual risk behaviors [22-24]. Parental monitoring has been identified as a protective factor because it is a process of supervising children, even when they are outside home, and minimizes adolescents' involvement in high risk peer groups [9,10,25-27]. Living with both parents is found to be protective against risky behaviors in most studies across countries [17, 28-29]. In terms of parental education, some studies report an inverse relationship between early SI and parental education or income, while others have not found such a relationship [30].

Adolescent variables: Although several factors may influence early sexual initiation in adolescents, this study focused on Spirituality, academic grades, ad self-esteem. Spirituality has been noted in the Juduo -Christian and Muslim cultures to be a proactive factor against high risk behaviors including early SI [31-34]. Parents who are spiritual can influence their children by providing a model of how spiritual beliefs and practices are important for coping with difficult life situations and for creating pro-social values in the community. A recent study [19] found that the spirituality of parents and children was protective against thigh risk behaviors sin $13-14$ year old Thai children. High academic grades appear to deter children from high risk behaviors. Most studies note that children with higher academic achievement and bonding with the school are less likely to engage in early sexual debut (Denny et al. 2011; Jovic et al. 2104) [35,36]. Self-esteem plays an important role in the field of mental health and social behavior. Several studies Lejuez et al., $2004[37,38]$ support the link between low self-esteem and early SI. Large cross sectional studies from different countries note that low self-esteem predicts both early onset of sexual activity and unprotected sex $[38,39]$. In contrast, Wheeler, [36] found that higher self-esteem at baseline had no effect on sexual debut for females while Spencer et al., [40] noted that the probability of having sex was linked with high selfesteem in boys but with low self-esteem in girls.

Other demographic variables obtained were gender and age, since these factors were noted in earlier research to affect early SI or risky behaviors in adolescents [17,41]. Gender differences are especially important as adolescent sexual activity is influenced by the biological and cultural nature of sexual behavior [17,42]. While earlier studies note higher rates of early SI among males this trend is changing as adolescent girls in Western societies are beginning to surpass boys in experimenting with sexual behavior.

Despite the rich research related to early sexual initiation in adolescents, there are some research gaps that should be addressed. Mostly studies have been conducted among adolescents in the western culture with Judeo-Christian traditions, with limited research focusing on early sexual initiation among adolescents in Eastern cultures, especially in Thailand. In addition, limited studies have looked at whether the Buddhist religion has a similar protective effect on early SI as it does in other religions. The objectives of this study were to identify the relationship among gender, self-esteem, family structure, parents' education, parental monitoring, school performance, spirituality and early SI among young Thai adolescents. Early SI was defined as engaging in sexual intercourse before the age of 15 years. The three research questions addressed were:
1) What is the prevalence of early SI in Thai adolescent males and females and what is the age for early SI?

2) What are the factors; parents' education, parental monitoring, school performance, and adolescent's gender, age, selfesteem, spirituality, that affect and early SI among Thai adolescents' and,

3) What factors are associated early SI_among Thai children?

\section{Methods}

Design: A cross sectional design with a survey method was used.

Setting: The data was collected in a secondary school in Nakhon Pathom in Thailand. Thailand is a Buddhist society with $94.6 \%$ of the population being Buddhist, 4.1\% Muslim and 1\% Christian [43].

Subjects: The total number of subjects in this study included a total of 430 Thai female and male students between the ages of 14 and 17 years in grades 9 through12. Convenience sampling was used to reach the appropriate number of subjects. A study sample of 400 was considered sufficient to detect a medium effect in the regression analysis with alpha set at 0.05 , and beta at 0.20 (power of 0.80 ).

Data collection: The principal investigator (PI) contacted the schools' administrator, explained the study and obtained his approval to conduct the study. All students $(\mathrm{N}=624)$ at the selected school and appropriate grades were invited to participate by sending letters to their parents briefing them about the study with consent and assent forms. Their approval to enroll their child in the study was obtained by having them sign the consent form and for the child to sign the assent form and returning it to the school administrators' office. The response rate was $69 \%(\mathrm{~N}=430)$. Efforts were made to prevent any disruption of daily academic activities, thus, the data was collected during recess or during art or gymnastic sessions and based on the school administrator's discretion. Children were asked to fill out the questionnaires with no identifying information. Children were advised about the importance of being honest in their responses to the questionnaires. The completion of the questionnaires was supervised by the PI and the teachers of the class, if a child needed a clarification about a question the PI was available to provide an explanation.

Instruments: All instruments were translated into Thai and then back translated into English following international guidelines for translation, a bilingual person translated the survey from English to Thai then a second bilingual person who had not seen the original English language version translated it back into Thai [44]. The bilingual translators were nurses with 10 to 15 years of nursing background experience.

Early SI was measured by self-report where students responded to two questions:

1) "Have you ever had sexual intercourse?" $0=$ No, $1=$ Yes.

2) If yes, what age did you have first sexual initiation

Parental monitoring: was assessed using the Parental Monitoring Scale (PMS), a 7-item scale which obtained information on the perception children had of the accuracy of their parents' knowledge of their activities, whereabouts and friends. Response options were $1=$ "never," $2=$ "rarely," $3=$ "sometimes," $4=$ "most of the time" and $5=$ "always". A higher score indicated a higher level of perceived parental monitoring [45]. The internal consistency for this study as per Cronbach's alpha was $\mathrm{r}=0.89$. 
Self -esteem: The Rosenberg Self- esteem scale was used which is a 10-item questionnaire with a 4-point scale is from strongly agree $(\mathrm{SA}=4)$ to strongly disagree $(\mathrm{SD}=1)$. The scores range between 10 and 40 with higher scores indicating higher self-esteem. The Cronbachs alpha for this study was $r=.87$.

Spirituality: The Daily Spiritual Experience Scale (DSES) was used, which is an instrument to measure spirituality. The DSES has been translated to over 20 languages and used in a diversity of cultures [46]. The questionnaire consists of 16 items; the first 15 items are scored on a Likert rating scale from 1 to $6: 6=$ never, and $1=$ many times a day and the 16th item has four response categories: $4=$ not at all, $3=$ somewhat close, $2=$ very close and $1=$ as close as possible. Although the DSES has been used in to assess spirituality in different religions, the word "God" was translated to a "higher power" as the Buddhist religion does not have the word God. In this current study, the internal consistency of the scale was high (Cranach's alpha, $r=0.97$ ).

Other demographic information obtained was gender, age and fathers highest eduacinal level.

Data analysis: Demographic characteristics were first analyzed using descriptive statistics; means and Standard deviations (sds) were used for continuous variables while numbers and percentages were used for categorical variables. Prevalence of early SI among genders was calculated as the the number of boys and girls who had early $\mathrm{Si}$ divided by the total number. An independent t-test or ANOVA was used to examine the effect of self-esteem, parents' education, parental monitoring, academic performance and spirituality on early SI, while chi-square tested the association between gender, family structure and early SI. A logistic regression was used to investigate the associations between the study variables and early SI. The Hosmer and Lemeshow test assessed the goodness of fit of the model.

Ethical approval was obtained from the following: Azusa Pacific University Institutional Review Board, the Christian University of Thailand Institution Review Board, and approval from the director of the secondary school in Nakhon Pathom, Thailand

\section{Results}

Table 1 provides the characteristics of the sample which included 430 children, $100 \%$ were Buddhists, of whom $53 \%$ were boys $(n=228)$, and $47 \%$ were girls $(n=202)$. Most of the participants lived with both parents $(77.4 \%)$, while $19.3 \%$ lived with a single parent and $8.1 \%$ lived with others. Almost $50 \%$ of the participants indicated that their parents had completed high school, followed by $26.9 \%$ who had elementary school education and $25.6 . \%$ who had a university degree. The ages of students ranged between 14 to19 years old (mean=16.24 \pm 4.21 ). Almost half the sample had a GPA between $3.01-4.00,47.7 \%$ had a GPA between $2.01-3.00$ and $6.1 \%$ had a GPA between 1.01-2.00.

\section{Answers to research questions}

Research question 1: What is the prevalence of early SI in Thai adolescent males and females and what is the age for early SI? The prevalence of early SI among males was $28.5 \%$, and for females it was $20.3 \%$. Eight percent of all participants reported having their first sexual experience between $11-13$ years, $60 \%$ reported early SI between $14-15$ years followed by $29 \%$ at 16 years. The youngest age reported by a male was 11 years and in girls it was 12 years. The average age was 14.84 years for males $( \pm 1.12)$ and 15.17 years for females $( \pm 1.67)$ which was not significantly different $(\mathrm{t}=-1.12, p>0.05)$.
Research question 2: What are the relationships between parents' education, parental monitoring, school performance, and adolescent's gender, self-esteem, spirituality, and early SI among Thai adolescents?

As seen in Table 2, all the variables except for age, were significantly associated with early SI. Males were more likely to engage in early sexual

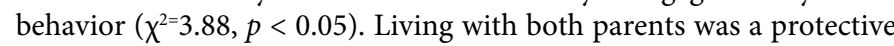
factor compared to living with one parent or with others $\left(\chi^{2}=25.59, p<\right.$ $.001)$. Higher paternal education was related to early $\mathrm{SI}(\mathrm{t}=3.32, \mathrm{df}=428$, $p<0.001$ ), where the mean was 24.20 ( \pm 5.45 ), for children who had not initiated sexual behavior compared to a mean of $16.38( \pm 5.37)$ for students who had engaged in early sexual behavior. Students with lower GPAs had initiated sex earlier compared to those with higher GPAs $(\mathrm{t}=6.8, p<0.001)$ with a mean of $2.62 \pm .53$ for children who had early SI compared to a mean of $3.03( \pm .52)$ for children who had not initiated sex early. Students with higher scores on spirituality were less likely to be involved in early sexual initiation $\mathrm{t}=-4.87, p<0.001,(56.26$ \pm 10.76 versus $48.60 \pm 16.16)$ and students with higher scores on selfesteem were less likely to be involvement in early sexual behavior than children with low self-esteem, $\mathrm{t}=4.94$, df. $=255.67, p<.001(2 \pm 5.37 \pm$ 4.74 versus $23.31 \pm 3.31$ ).

Research question 3: What factors are associated early SI among Thai children?'

As shown in Table 3, the overall model was significant; the variance accounted in the model was $22 \%$. The Hosmer and Lemeshow goodness of fit showed that the model was a good fit $\left(\chi^{2=10.48, ~} \mathrm{df}=8\right.$, $p=.233$ ). The variables that were significantly associated with early SI were; parental monitoring, family structure, grades, and spirituality. Parental monitoring was the most significant predictor for early SI with an odds ratio of 0.23 , indicating that higher scores on parental monitoring decreased the likelihood of early SI, $(\beta=-1.55, \mathrm{OR}=0.23$, 95\% CI $=0.138-0.324, p<0.01$ ). Family structure was the second significant variable associated with early SI, whereby children who lived with both parents had less odds of initiation in early sexual behaviour, $(\beta=0.97, \mathrm{OR}=0.45, \mathrm{CI}=0.213-0.666, p<0.01)$. Grades were the third variable significantly associated with early SI whereby children with higher grades were less likely to engage in early sexual behavior $(\beta=$ $.78, \mathrm{OR}=0.57, \mathrm{CI}=0.138-0.324, p<0.05)$. Spirituality was the fourth variable significantly associated with early SI where higher scores on the DSES were protective against early SI, $(\beta=-.66, \mathrm{OR}=0.67, \mathrm{CI}=0.315$ $0.842, p<0.05)$.

\section{Discussiona}

This is one of few studies that assessed spiritually as a possible predictor among other variables of early SI in Thai children. The findings note that $60 \%$ of children reported early SI at ages between 14-15 years old which is slightly higher than reported by the last survey by the national sexual surveillance system in Thailand (National AIDS Prevention and Alleviation Committee in Thailand, 2010) and could be explained by the fact that this study setting was a private school in a suburb as opposed to data collected in the city of Bangkok, generally. Boys were more likely to engage in early sexual behaviors which is an expected finding and supported by earlier studies [15,17]. Thai girls may be less likely to be involved in early sexual activity due to gender norms that are still prevalent in Thailand, which favour females remaining virgins until marriage [27,47]. It is worth noting that while the prevalence of Early SI was higher for boys compared to girls, the average age of early SI was not significantly different indicating a trend for both genders to initiate sex at an earlier age. 
Higher scores on self- esteem, higher grades, living with both parents, and higher parental education were all protective against early SI which is supported by earlier studies [37-39]. What is new in this study is that higher spiritually was also a protective factor which is supported by findings with children from Christian, Muslim and Jewish faiths [31-34] but has not been previously reported with the Buddhist religion. It is worth noting that a study by Chamratrithirong et al., [19] found that spirituality in parents and children was associatedwith less intention to have sex in Thai adolescents. In this latter study the authors argued that the Buddhist teachings and the related parental sense of duty increased monitoring practices, which in turn could prevent children from engaging in problem behaviors. Likewise a study in Korea found that males who attended religious services at least once a week were less likely to initiate sex at an earlier age compared to males who never attended religious services and women who prayed at least once a day, were also less likely to be initiate early sex compared to those who never prayed [48].

Although all the variables assessed in this study except for age, were associated with early SI, in the logistic regression only four variables (parental monitoring, family structure, grades and spirituality) were statistically significant predictors of SI. Gender, self-esteem, parents' education were not statistically significant predictors. It has been well established that when parents are involved in tracking the whereabouts and doings of their offspring, risky behaviors are less likely to [25,49]. Family structure also plays an important role in protecting children from risky behaviors which has been supported by several earlier studies. Living with both parents as noted in this study was protective factor against early SI which has also been documented in several studies across countries $[9,17,29,32]$. Academic performance decreases the odds of early SI which is consistent with several previous studies. For example, Schvaneveldt et al., [50] found that there was a bidirectional relaionship between educational goals, academic achievement, and early SI. Adolescents who have high education goals and academic achievement may be more likely to delay having sex because of their knowedge of the negative consequences of early SI, which might affect their future goals. Similar to most studeis in the West we found that Spirituailty was a protecive factor agnaist Early SI. The association between adolescents who are spiritual and decreased risky behaviours has been well supported in the literature [31,51-53].

\section{Clinical implications}

The importance of family monitoring and spirituality in preventing early SI may need to be reinforced to improve the health of young adolescents. The value of this study is the presentation of evidence demonstrating that the Buddhist religion is a protective factor and plays an important role in positive health behavior choices. Thus, it is important for health providers to examine factors that affect decision making in early adolescents. The public health message should emphasize the importance of family and spiritual factors that can reduce the incidence of risky behaviours and the resulting health problems. In Light of this study, it is important for clinicians to encourage parents to discuss with their children sexual issues such as STIs, menstruation, first spontaneous ejaculation and how pregnancy occurs, all of which can prevent early SI.

\section{Limitations}

Despite the strengths of this study and the interesting addition of Buddihst spirituality as a factor that can influence early SI, the study has several limitations. First, it was a cross-sectional study which precludes any assumptions of causality. Second, self-report and recall may not be provide accurate information as respondents may forget or omit answers. In Addition, many children may not have been honest in their responses as sexual behavior is a sensitive subject, and discussions about sex are generally socially unacceptable in the culture of Thailand. Although the surveys were anonymous and the PI assured the parents and children that their responses were confidential bias in reporting may still have occurred. Future research on early sexual initiation may benefit from the inclusion of variables that were not available in this study such as peer relationships, parental-child discussions about sex, and partner age.

\section{Conclusion}

The results of this study will provide additional grounds for interventions that may help prevent early SI in Thai adolescents. The benefits may extend not only to Thai adolescents but to children living in comparable cultures in the Asian world and who may be experiencing similar high rates of early SI. Interventions may need to be implemented earlier than the age of first SI if they are to be effective preventive measures. In addition, the importance of spiritual beliefs and practices that have traditionally sustained the culture of Thailand need to be emphasized and strengthened in prevention programs as they are important determinants in the prevent problem behaviors among Thai adolescents.

\section{References}

1. Reese BM, Haydon AA, Herring AH, Halpern CT (2013) The association between sequences of sexual initiation and the likelihood of teenage pregnancy. $J$ Adolesc Health 52: 228-233. [Crossref]

2. Iacono WG, Malone SM, McGue M (2008) Behavioraldisinhibition and the development of early-onset addiction: Common and specific influences. Annual Review of Clinical Psychology 325-348.

3. Kaestle CE, Halpern CT, Miller WC, Ford C (2005) Young age at first sexual intercourse and sexually transmitted infections in adolescents and young adults. American Journal of Epidemiology 161: 774-780.

4. Buhi ER, Goodson P (2007) Predictors of adolescent sexual behavior and intention: a theory-guided systematic review. J Adolesc Health 40: 4-21. [Crossref]

5. Sneed CD (2009) Sexual risk behavior among early initiators of sexual intercourse. AIDS Care 21: 1395-1400. [Crossref]

6. Reese BM, Choukas-Bradley S, Herring AH, Halpern CT (2014) Correlates of Adolescent and Young Adult Sexual?Initiation Patterns. Perspectives in Sex \& Reproductive Health 46: 211-221.

7. Cavazos-Rehg PA, Krauss MJ, Spitznagel EL, Schootman M, Bucholz KK, et al. (2009) Age of sexual debut among US adolescents. Contraception 80: 158-162. [Crossref]

8. Centers for Disease Control \& Prevention (2012a). Youth risk behavior surveillanceUnited States, 2011.MMWR Surveillance Summaries 61(SS-04) 1162.

9. Lenciauskiene I, Zaborskis A (2008) The effects of family structure, parent-child relationship and parental monitoring on early sexual behaviour among adolescents in nine European countries. Scand J Public Health 36: 607-618. [Crossref]

10. Madkour AS, Farhat T, Halpern CT, Godeau E, Gabhainn SN (2010) Early adolescent sexual initiation as a problem behavior: a comparative study of five nations. J Adolesc Health 47: 389-398. [Crossref]

11. ChartsBin statistics collector team (2009) Average Age at First sex by Country ChartsBin.com, Retrieved November, 201.

12. Thailand - 2010 Country Progress Report National AIDS Prevention and Alleviation Committee. Accessed Nov, 2014.

13. Khumsaen N, Gary FA (2009) Determinants of actual condom use among adolescents in Thailand. The Journal of the Association of Nurses in AIDS Care 20: 218-219.

14. Rasamimari A, Dancy B, Smith J (2008) HIV risk behaviours and situations as perceived by Thai adolescent daughters and their mothers in Bangkok, Thailand. AIDS Care 20: 181-187. [Crossref] 
15. Techasrivichien T, Darawuttimaprakorn N, Punpuing S, Musumari PM, Lukhele BW, et al. (2014) Changes in Sexual Behavior and Attitudes Across Generations and Gender Among a Population-Based Probability Sample From an Urbanizing Province in Thailand. Archives of Sexual Behavoir. [Epub ahead of print].

16. Bureau of Epidemiology (2013) sexually transmitted infections. Annual epidemiological surveillance report 2012 103-105.

17. Liu A, Kilmarx P, Jenkins RA, Manopaiboon C, Mock PA, et al. (2006) Sexual initiation, substance use, and sexual behavior and knowledge among vocational students in northern Thailand. Internationl Family Plannin Perspectives32: 126-135.

18. Sasson VR (2014) Buddhism and children: scholars struggle with the story of the Buddha's son. Child Abuse Negl 38: 593-599. [Crossref]

19. Chamratrithirong A, Miller BA, Byrnes HF, Rhucharoenpornpanich O, Cupp PK, et al. (2010) Spirituality within the family and the prevention of health risk behavior among adolescents in Bangkok, Thailand. SocSci Med 71: 1855-1863. [Crossref]

20. Chen AC, Thompson EA, Morrison-Beedy D (2010) Multi-system influences on adolescent risky sexual behavior. Res Nurs Health 33: 512-527. [Crossref]

21. Bronfenbrenner U (1977) Toward an experimental ecology of human development. American Psychologist513-531.

22. Malcolm S, Huang S, Cordova D, Freitas D, Arzon M, et al. (2013) Predicting condom use attitudes, norms, and control beliefs in Hispanic problem behavior youth: the effects of family functioning and parent-adolescent communication about sex on condom use. Health Education and Behavior 40: 384-391.

23. Sandler IN, Schoenfelder EN, Wolchik SA, MacKinnon DP (2011) Long-term impact of prevention programs to promote effective parenting: lasting effects but uncertain processes. Annu Rev Psychol 62: 299-329.

24. Szapocznik J, Prado G, Burlew AK, Williams RA, Santisteban DA (2007) Drug abuse in African American and Hispanic adolescents: culture, development, and behavior. Annu Rev ClinPsychol 3: 77-105. [Crossref]

25. Ahmadi K, KhodadadiSangdeh J, Aminimanesh S, Mollazamani A, Khanzade M, et al. (2013) The role of parental monitoring and affiliation with deviant peers in adolescents' sexual risk taking: toward an interactional model. International Journal of High Risk Behavior and Addiction 2: 22-27.

26. Dessie Y, Berhane Y, Worku A3 (2014) High parental monitoring prevents adolescents from engaging in risky sexual practices in Harar, Ethiopia. Glob Health Action 7: 25724. [Crossref]

27. Rhucharoenpornpanich O, Chamratrithirong A, Fongkaew W, Rosati MJ, Miller BA, et al. (2010) Parenting and adolescent problem behaviors: a comparative study of sons and daughters in Thailand. J Med Assoc Thai 93: 293-300. [Crossref]

28. Bailey JA, Fleming CB, Henson JN, Catalano RF, Haggerty KP (2008) Sexual risk behavior 6 months post-high school: associations with college attendance, living with a parent, and prior risk behavior. J Adolesc Health 42: 573-579. [Crossref]

29. Pilgrim NA, Ahmed S, Gray RH, Sekasanvu J, Lutalo T, et al. (2014) Family structure effects on early sexual debut among adolescent girls in Rakai, Uganda. Vulnerable Child Youth Studies 9: 193-205.

30. Tangmunkongvorakul A, Carmichael G, Banwell C, Seubsman SA, Sleigh A (2012) Coital experience among adolescents in three social-educational groups in urban Chiang Mai, Thailand. Asian Population Studies 8: 39-63.

31. Cotton S, Berry D (2007) Religiosity, spirituality, and adolescent sexuality. Adolesc Med State Art Rev 18: 471-488. [Crossref]

32. Haglund KA, Fehring RJ (2010) The association of religiosity, sexual education, and parental factors with risky sexual behaviors among adolescents and young adults. $J$ Relig Health 49: 460-472. [Crossref]

33. Koenig H , George L Chen, J Hays, J Larson J, Blazer D (1998) The relationship between religious activities and cigarette smoking in older adults. Journal of Gerontology: Medical Science 53: 426-434.
34. Sinha JW, Cnaan RA, Gelles RJ (2007) Adolescent risk behaviors and religion: findings from a national study. $J$ Adolesc 30: 231-249. [Crossref]

35. Laflin MT, Wang J, Barry M (2008) A longitudinal study of adolescent transition from virgin to nonvirgin status. J Adolesc Health 42: 228-236. [Crossref]

36. Wheeler SB (2010) Effects of self-esteem and academic performance on adolescent decision-making: an examination of early sexual intercourse and illegal substance use. $J$ Adolesc Health 47: 582-590. [Crossref]

37. Kalina O, Geckova AM, Klein D, Jarcuska P, Orosova O, et al. (2011) Psychosocial factors associated with sexual behavior in early adolescence. EuopeanJouranl of ontraceptive and Reproductive Health Care 16: 298-306.

38. Wild LG, Flisher AJ, Bhana A, Lombard C (2004) Associations among adolescent risk behaviours and self-esteem in six domains. J Child Psychol Psychiatry 45: 1454-1467. [Crossref]

39. Magnani RJ, Seiber EE, Gutierrez EZ, Vereau D (2001) Correlates of sexual activity and condom use among secondary-school students in urban Peru. Stud FamPlann 32: 53-66. [Crossref]

40. Spencer JM, Zimet GD, Aalsma MC, Orr DP (2002) Self-esteem as a predictor of initiation of coitus in early adolescents. Pediatrics 109: 581-584. [Crossref]

41. Rada C (2014) Sexual behaviour and sexual and reproductive health education: a crosssectional study in Romania. Reprod Health 11: 48. [Crossref]

42. O'Donnell L, Myint-U A, O'Donnell CR, Stueve A (2003) Long-term influence of sexual norms and attitudes on timing of sexual initiation among urban minority youth. $J$ Sch Health 73: 68-75. [Crossref]

43. The National Statistical Office, (Underwood). The National Statistical Office Preliminary report: The 2000 population and housing census. Ministry of Information and Communication Technology, Bangkok.

44. Bowden A, Fox-Rushb JA (2003) A systematic and critical review of the process of translation and adaptation of generic health-related quality of life measures in Africa, Asia, Eastern Europe, the Middle East, South America. Social Science and Medicine Journal 57: 1289-12306.

45. Li X, Stanton B, Feigelman S (2000) Impact of perceived parental monitoring on adolescent risk behavior over 4 years. $J$ Adolesc Health 27: 49-56. [Crossref]

46. Underwood LG, Teresi JA (2002)The daily spiritual experience scale: development, theoretical description, reliability, exploratory factor analysis, and preliminary construct validity using health-related data. Annals of Behavioral Medicine 24: 22-33.

47. Supametaporn P, Stern PN, Rodcumdee B, Chaiyawat W (2010) Waiting for the right time: how and why young Thai women manage to avoid heterosexual intercourse. Health Care Women Int 31: 737-754. [Crossref]

48. Kang PP, Romo LF (2011) The role of religious involvement on depression, risky behavior, and academic performance among Korean American adolescents. J Adolesc 34: 767-778.[Crossref]

49. Madkour AS, Farhat T, Halpern CT, Gabhainn SN, Godeau E (2012) Parents' support and knowledge of their daughters' lives, and females' early sexual initiation in nine European countries. Perspectives on Sexual and Reproductive Health 44: 167-175.

50. Schvaneveldt PL, Miller BC, Berry EH, Lee TR (2001) Academic goals, achievement, and age at first sexual intercourse: longitudinal, bidirectional influences. Adolescence 36: 767-787.

51. Rostosky SS, Regnerus MD, Wright ML (2003) Coital debut: the role of religiosity and sex attitudes in the Add Health Survey. J Sex Res 40: 358-367. [Crossref]

52. Kirby DB (2008)The impact of abstinence and comprehensive sex and STD/HIV education programs on adolescent sexual behavior. Sexuality Research and Social Policy 5: 18-27.

53. Ma Q, Ono-Kihara M, Cong L, Xu G, Pan X, et al. (2009) Early initiation of sexua activity: a risk factor for sexually transmitted diseases, HIV infection, and unwanted pregnancy among university students in China. BMC Public Health 9: 111.

Copyright: (C2016 Sasitorn Roojanavech RN. This is an open-access article distributed under the terms of the Creative Commons Attribution License, which permits unrestricted use, distribution, and reproduction in any medium, provided the original author and source are credited. 